\title{
Abordagens para Avaliação de Softwares Educativos e sua Coerência com os Modelos de Qualidade de Software
}

\author{
Ozonias de O. B. Junior ${ }^{1}$, Yuska P. C. Aguiar', Tatiana A. Tavares ${ }^{3}$ \\ ${ }^{1}$ Centro de Informática - Universidade Federal da Paraíba (UFPB) - João Pessoa, PB - \\ Brasil \\ ${ }^{2}$ Departamento de Ciências Exatas - Universidade Federal da Paraíba (UFPB) - Rio \\ Tinto - PB - Brasil \\ ${ }^{1,3}$ Centro de Desenvolvimento Tecnológico - CDTec - Universidade Federal de Pelotas \\ (UFPEL) - Pelotas, RS - Brasil \\ ozonias.oliveira@ppgi.ci.ufpb.br; yuska@dcx.ufpb.br, \\ tatianalinf.ufpel.edu.br
}

\begin{abstract}
This article describes the analysis of 14 approaches to evaluation of Educational Software (SE) held in order to identify the coherence and comprehensiveness of such procedures in relation to established models of classical literature for Software Quality (ISO 9126-1 and SWEBOK Guide), Quality of Service (ISO 9241-1 and Heuristics Usability) and the Precepts of Education. The mapping was developed due to the large divergence and differences between the approaches, by carrying out an initial analysis of them, in order to make the evaluation experiment more formal and less subjective. The result obtained shows the need for standardization of approaches to SE evaluation.
\end{abstract}

Resumo. Este artigo descreve a análise de 14 abordagens para avaliação de Softwares Educativos (SE), realizada com o objetivo de identificar a coerência e abrangência de tais procedimentos em relação aos modelos consagrados da literatura clássica para a Qualidade de Software (Norma ISO 9126-1 e Swebok Guide), Qualidade de Uso (Norma ISO 9241-1 e Heurísticas de Usabilidade) e os Preceitos da Pedagogia. O mapeamento foi elaborado devido à grande divergência e diferenças entre as abordagens, realizando uma análise inicial das mesmas, a fim de tornar os experimentos de avaliação mais formais e menos subjetivos. O resultado alcançado revela a necessidade de padronização das abordagens para avaliação de SE.

\section{Introdução}

As Tecnologias de Informação e Comunicação (TIC) estão presentes nas atividades humanas, sendo expressiva o seu uso no âmbito educacional a fim de aproximar os indivíduos com as novas tecnologias [De França e Da Silva 2014] a partir do uso de diversos recursos de informática dentro e fora da sala de aula.

A combinação de Educação e Tecnologia favorece a qualidade do processo de ensino-aprendizagem, afetando métodos, práticas e recursos de ensino [Coomans e Lacerda 2015]. O uso correto desses recursos propicia o aprendizado lúdico; melhora a interpretação do conteúdo teórico a partir da prática; exercita a resolução de problemas em tempo ágil; possibilita o aprendizado a partir dos erros; e reduz a passividade dos 
V Congresso Brasileiro de Informática na Educação (CBIE 2016)

Anais do XXVII Simpósio Brasileiro de Informática na Educação (SBIE 2016)

alunos. Portanto, a prática pedagógica se beneficia com o uso de recursos multimídia, elevando o potencial de abordagens tradicionais.

Vale ressaltar que estes recursos educacionais devem ser adequados pedagogicamente aos objetivos estabelecidos pelos professores, corretos funcionalmente de acordo com a Engenharia de Software e direcionados para as características, necessidades e habilidades de seus usuários, segundo a Engenharia da Usabilidade. Inadequações nestas vertentes podem comprometer a potencialidade do recurso e prejudicar seu propósito de auxiliar o processo de ensino-aprendizagem. Logo, se faz necessário avaliar estes recursos frente à sua adequação ao contexto de aplicação pretendido [Da Silva e De França 2014].

A literatura contempla vários trabalhos sobre o uso de TICs na educação, sendo significativa a quantidade de publicações sobre a avaliação e uso de Softwares Educativos (SEs) como facilitador no processo de ensino-aprendizagem. Considerando abordagens utilizadas na avaliação de SE, é possível identificar uma gama de proposições [Muchielli 1987; Reeves 1994; Gladcheff 2001; Silva 2002; Atayde 2003; Webber et al. 2009; Savi 2010; Coomans 2015]. Esta diversidade indica a relevância do tema e leva à reflexão sobre a falta de consenso para avaliação de SEs.

Acredita-se que o início da padronização para avaliação de SE consiste na análise das abordagens existentes para identificar suas características em termos de (i) natureza da avaliação (objetiva, subjetiva ou mista); (ii) aspectos de qualidade avaliados (pedagógicos, de software e de uso); (iii) instrumentos para coleta de dados (quantidade de critérios; tipos de escalas); e (iv) resultados (diagnóstico) obtidos. A partir de então é essencial compreender como estas abordagens referem-se aos modelos de Qualidade de Software (QS) [ISO 9126-1 2003; Swebok 2003], Qualidade de Uso (QU) [ISO 9241-1 2003; Bastien e Scapin 1993; Nielsen 1994; Shneiderman e Plaisant 2004] e aos preceitos da pedagogia (Qualidade Pedagógica - QP) [Oliveira et al 2001; Silva 2012]. Portanto, um mapeamento das abordagens propostas para avaliação de SE com os modelos de qualidade é útil para conhecer a coerência e abrangência entre estes, com o objetivo de responder as questões de pesquisa: Quantos e quais critérios de cada abordagem correspondem aos critérios definidos pelos modelos de referências (QS QU e QP)? Quantos e quais dos critérios dos modelos de referência ( $Q S Q U$ e QP) não foram contemplados por nenhuma das abordagens?

A qualidade de software consiste na conformidade com requisitos funcionais e de desempenho explicitamente declarados [Pressman 1997], sendo a qualidade de software classificada em interna (a qualidade que está embutida no software), externa (que é referenciada na parte exterior do software) e de uso (referente ao momento de utilização do software pelo usuário) [Swebook 2004]. A qualidade pedagógica é definida como a capacidade de viabilizar o uso de SE no contexto educativo, incluindo a verificação da didática e da teoria pedagógica embutida no software [Silva 2012].

Este artigo considera a análise, sob a ótica citada acima, das 14 abordagens para avaliação de SEs: Técnica de Muchielli [Muchielli 1987], Método Rocha [Rocha et al. 1993], MAQSE [Campos 1994], Método de Reeves [Reeves 1994], TICESE [Gamez 1998], Método ASE [Vieira 1999], IAQSEM [Gladcheff 2001], MAEP [Silva 2002], LORI [Nesbit et al. 2003], MAQSEI [Atayde 2003], FASE [Webber et al. 2009], Modelo de Savi [Savi 2010], Instrumento de Rodrigues [Rodrigues 2014] e PETESE [Coomans et al. 2015]. 
V Congresso Brasileiro de Informática na Educação (CBIE 2016)

Anais do XXVII Simpósio Brasileiro de Informática na Educação (SBIE 2016)

\section{Avaliação de Softwares Educativos}

Da Silva et al (2011) propõe uma lista de 50 critérios para avaliação de SE que compila três outros trabalhos [Oliveira et al 2001; Atayde 2003; Webber 2009]. Como resultado, os autores indicaram que os critérios propostos para avaliação de SE não contemplam todos os aspectos que podem ser vislumbrados em avaliações dessa natureza, e também mencionaram que existem distintas maneiras para lidar com os apanhados (análises dos critérios) das avaliações, podendo levar à interpretações dúbias.

Godoi e Padovani (2011) apresentam a análise de um estudo analítico sobre 23 instrumentos avaliativos de materiais didáticos digitais, dos quais 7 (MAQSEI, TICESE, Reeves, MAQSE, ASE, MAEP e IAQSEM) são contemplados neste estudo. Das 23 abordagens (ou instrumentos avaliativos), 7 foram aplicadas e o resultado indica como pontos positivos: a clareza na sua aplicação, a clareza na geração dos resultados e a objetividade das abordagens. Como pontos negativos são descritos o número elevado de critérios para avaliação (para uma das abordagens com 36 critérios), dificultando a avaliação e tornando-a cansativa, e a dificuldade na interpretação de termos técnicos (critérios). Como resultado, indicaram a necessidade de agrupar os critérios em ergonômicos e pedagógicos, assim como reduzir a quantidade total de critérios avaliados.

O trabalho de Oliveira e Aguiar (2014) apresenta a comparação de 8 abordagens (6 contempladas nesta pesquisa: Rocha, TICESE, LORI, Muchielli, Reeves e MAQSE) de avaliação de SE a fim de identificar qual destas seria mais completa, em termos dos critérios adotados, para avaliar aspectos pedagógicos, de interface e técnicos. Como resultado, indicam que a aplicação isolada de qualquer uma das abordagens resulta em um diagnóstico que desconsidera critérios relevantes, propondo, portanto, a combinação das abordagens de forma que sua aplicação conjunta amplie a abrangência dos resultados obtidos.

Peixoto et al. (2015) apresentam uma Revisão Sistemática da Literatura sobre abordagens para avaliação de jogos educacionais multiusuários. Nestes os autores identificaram os instrumentos para coleta de dados das abordagens, as dimensões avaliadas e os resultados de satisfação sobre as avaliações realizadas. Como resultado, concluíram que as avaliações deste tipo de SE estão restritas a testes-piloto de pequeno porte e/ou avaliações subjetivas. Experimentos formais não são utilizados para avaliar a eficácia dos SEs no processo de ensino-aprendizagem.

Os trabalhos citados apresentam algumas limitações à análise realizada nesta pesquisa, a saber: (i) as abordagens foram analisadas com base em sua aplicação, sem que os procedimentos metodológicos fossem descritos com detalhes, não havendo, ainda, uma investigação sobre os critérios sugeridos nas abordagens de avaliação de SE e o referencial teórico adotado na literatura para a qualidade de software, de uso ou preceitos pedagógicos; (ii) a análise acerca de instrumentos avaliativos de materiais didáticos (distintos tipos de recursos didáticos), não específicos para a avaliação de SE. Este trabalho tem como diferencial a análise comparativa entre 14 abordagens para avaliação de SE, um mapeamento entre os critérios contemplados nestas abordagens com relação aos modelos clássicos de qualidade de software, de uso e de preceitos da pedagogia. Além disso, busca-se a padronização dos termos utilizados para descrever os critérios de avaliação, em prol de uma sistematização que permita tornar os experimentos de avaliação menos subjetivos e mais formais [Peixoto et al. 2015]. 
V Congresso Brasileiro de Informática na Educação (CBIE 2016)

Anais do XXVII Simpósio Brasileiro de Informática na Educação (SBIE 2016)

\section{Metodologia da Pesquisa}

A metodologia adotada é de natureza aplicada, com abordagem quantitativa e qualitativa do problema, tendo objetivo exploratório a partir de pesquisa bibliográfica. A seleção das abordagens consideradas neste trabalho de pesquisa foi precedida de uma pesquisa bibliográfica exploratória que compreendeu publicações de 2005 à 2015. A busca foi realizada em anais de eventos que possuem trilhas ou são destinados ao tema de informática na educação, a saber: Congresso Brasileiro de Informática na Educação (CBIE), Workshop de Informática na Escola (WIE), Congresso da Sociedade Brasileira de Computação (CSBC), Workshop sobre Educação em Computação (WEI), Revista Brasileira de Informática na Educação (RBIE), Revista Novas Tecnologias na Educação (RENOTE) e a Internacional Conference on Applied Human Factors on Ergonomics (AHFE). O foco desta pesquisa inicial foi de encontrar trabalhos que descrevessem a aplicação de abordagens para avaliação de SE.

O objetivo consistiu em identificar abordagens para avaliação de SE, assim como identificar possíveis dificuldades encontradas em sua aplicação. As buscas foram realizadas a partir da análise dos títulos dos trabalhos, resumos e palavras chaves. A seleção de artigos considerou a pertinência da publicação ao tema de interesse a partir dos termos de busca método, técnica, instrumento e abordagem para avaliação de SE. $\mathrm{Na}$ ocasião foram selecionados 62 artigos, e nestes um total de 12 abordagens eram citadas. Dentre as 14 abordagens consideradas neste trabalho de pesquisa, duas não foram encontradas no levantamento bibliográfico inicial: Instrumento de Rodrigues e PETESE. Este fato se dá por serem trabalhos recentes (2014 e 2015, respectivamente) e ainda não existirem publicações que relatem sua aplicação até a data de realização desta pesquisa. Portanto a descrição dos resultados parciais do levantamento bibliográfico contempla 12 das 14 abordagens estudadas por completo nesta pesquisa. A partir das leituras realizadas foi possível identificar uma diversidade em relação aos tipos de SE avaliados, assim como das respectivas áreas de ensino associadas. Identificou-se a avaliação de SE dos tipos de exercício e prática, multimídia e internet, jogo eletrônico e simulação. As áreas de ensino contempladas como alvo das avaliações nos artigos foram: matemática, computação, química, saúde, psicologia, português, geografia, história, biologia, física e pedagogia. Esta análise exploratória, além de permitir conhecer as abordagens selecionadas para este estudo, permitiu identificar relevância no tema de estudo e diversidade de tipos de SE que vêm sendo avaliados.

Para a análise inicial das 12 abordagens identificadas no levantamento bibliográfico, as respectivas publicações originais foram resgatadas para se conhecer a proposição a partir da fonte. Com acesso a este documento, o seu ano de publicação e a quantidade de citações feitas para este trabalho foram relacionados a fim de perceber a atualidade e relevância da publicação - é importante ressaltar que uma publicação antiga, porém com muitas citações atuais, é um forte indício de que esta é relevante para a comunidade científica e que continua em uso mesmo muito tempo depois de sua proposição. Na Tabela 1 têm-se, por coluna, a abordagem encontrada, seu ano de publicação original, o número de citações para o trabalho original, o tipo de SE (EP: Exercício e Prática; S: Simulação; MI: Multimídia e Internet; JE: Jogo Eletrônico) avaliado pela abordagem nos artigos que a citaram e as áreas de conhecimento dos SEs contemplados nas avaliações. 
V Congresso Brasileiro de Informática na Educação (CBIE 2016)

Anais do XXVII Simpósio Brasileiro de Informática na Educação (SBIE 2016)

Tabela 1. Aplicação das abordagens de avaliação de SE

\begin{tabular}{|c|c|c|c|c|c|c|c|}
\hline \multirow{2}{*}{ Abordagem } & \multirow{2}{*}{ Ano } & \multirow{2}{*}{$\begin{array}{c}\text { \# de } \\
\text { Citações }\end{array}$} & \multicolumn{4}{|c|}{ Tipo do SE Avaliado } & \multirow{2}{*}{ Áreas de Conhecimento } \\
\hline & & & EP & $\mathbf{S}$ & MI & $\mathbf{J E}$ & \\
\hline $\begin{array}{l}\text { Técnica de } \\
\text { Muchielli }\end{array}$ & 1987 & 2 & $\mathbf{x}$ & $\mathbf{x}$ & & & matemática e computação \\
\hline Método Rocha & 1993 & 4 & $\mathbf{x}$ & & & & matemática e português \\
\hline MAQSE & 1994 & 3 & $\mathbf{x}$ & $\mathbf{x}$ & $\mathbf{x}$ & & $\begin{array}{l}\text { matemática, português e } \\
\text { química }\end{array}$ \\
\hline $\begin{array}{l}\text { Método de } \\
\text { Reeves }\end{array}$ & 1994 & 6 & $\mathbf{x}$ & $\mathbf{x}$ & $\mathbf{x}$ & $\mathbf{x}$ & $\begin{array}{l}\text { matemática, saúde e } \\
\text { computação }\end{array}$ \\
\hline TICESE & 1998 & 4 & $\mathbf{x}$ & $\mathbf{x}$ & & $\mathbf{x}$ & matemática e português \\
\hline Método ASE & 1999 & 5 & $\mathbf{x}$ & $\mathbf{x}$ & & $\mathbf{x}$ & matemática e ciências \\
\hline IAQSEM & 2001 & 12 & $\mathbf{x}$ & $\mathbf{x}$ & & $\mathbf{x}$ & $\begin{array}{l}\text { português, matemática, } \\
\text { química e computação }\end{array}$ \\
\hline MAEP & 2002 & 3 & & & $\mathbf{x}$ & $\mathbf{x}$ & $\begin{array}{l}\text { português, computação e } \\
\text { engenharia civil }\end{array}$ \\
\hline LORI & 2003 & 4 & & & & $\mathbf{x}$ & matemática e computação \\
\hline MAQSEI & 2003 & 5 & & & & $\mathbf{x}$ & computação \\
\hline FASE & 2009 & 4 & & & & $\mathbf{x}$ & matemática e computação \\
\hline Modelo Savi & 2010 & 13 & $\mathbf{x}$ & $\mathbf{x}$ & & $\mathbf{x}$ & $\begin{array}{l}\text { matemática, computação, } \\
\text { física, português, história } \\
\text { e biologia }\end{array}$ \\
\hline
\end{tabular}

Uma vez selecionadas as abordagens, a caracterização destas foi realizada considerando os elementos: (i) natureza da avaliação (objetiva, subjetiva ou mista); (ii) aspectos de qualidade avaliados contemplados por seus critérios (pedagógicos, de software e de uso); (iii) instrumentos para coleta de dados (quantidade de critérios, tipos de escalas); e (iv) resultados (diagnóstico) obtidos. Os detalhes sobre a caracterização estão descritos na seção 4.1 deste artigo. Tendo conhecimento sobre as abordagens e suas características a terceira etapa da pesquisa foi realizada. Para o mapeamento das abordagens de acordo com os modelos de referência da literatura para Qualidade de Software (QS), Qualidade de Uso (QU) e preceitos de Qualidade Pedagógica (QP) foi elaborada uma planilha no formato Excel disponível no link: http://tinyurl.com/jn637ct.

Inicialmente identificou-se, para cada modelo, a existência de critérios repetidos. Para QS foram comparados os 27 critérios disponíveis na Norma ISO 9126-1 [2003] com os 11 do Swebok Guide [2003], dos quais 16 eram equivalentes, restando 21 critérios de Qualidade de Software como modelo de referência. Para QU foram comparados os 7 critérios disponíveis na Norma ISO 9241-1 [2003] com as 10 heurísticas definidas por Nielsen [1994], as 8 propostas por Shneiderman e Plaisant [2004] e as 8 sugeridas por Bastien e Scapin [1993]. O modelo de Qualidade de Uso, sem duplicações, passa a ser composto por 12 critérios. Para os preceitos pedagógicos de qualidade foram consideradas as propostas de Oliveira [2001] (19 critérios) e Silva [2012] (14 critérios), contemplando 27 critérios após a retirada dos duplicados. Os critérios duplicados foram alocados apenas em uma das categorias (mais pertinente). $\mathrm{O}$ total de 60 critérios é utilizado como referência neste trabalho de pesquisa.

Os critérios de cada abordagem foram correlacionados com àqueles dos modelos de qualidade adotados como referência. Como resultado, espera-se identificar a coerência entre as abordagens para avaliação de SE e os modelos de referência na literatura, assim como a abrangência de cada abordagem (Passo 3 da análise dos resultados). Os detalhes do mapeamento estão descritos na seção 4. 
V Congresso Brasileiro de Informática na Educação (CBIE 2016)

Anais do XXVII Simpósio Brasileiro de Informática na Educação (SBIE 2016)

\section{Análise das Abordagens para Avaliação de SE}

Considerando a natureza da avaliação, das 14 abordagens estudadas 7 são objetivas (Reeves, MAQSE, Muchielli, LORI, Rocha, MAEP e FASE), 2 são subjetivas (MAQSEI e IAQSEM) e 5 são mistas (TICESE, Savi, ASE, Rodrigues e PETESE). A natureza de avaliação mista considera a utilização de recursos objetivos e subjetivos para avaliar SE, contemplando listas de critérios, entrevistas e observação.

O Gráfico 1 apresenta o agrupamento dos critérios propostos pelas abordagens em qualidade de software, qualidade de uso e qualidade pedagógica. A partir deste percebe-se uma variação significativa na quantidade total de critérios sugeridos pelas abordagens 9 critérios para MAQSE e LORI e 68 para PETESE. Um número baixo de critérios pode induzir o pensamento de facilidade de aplicação da abordagem, no entanto, geralmente estão associados a altos níveis de abstração, o que pode tornar seu uso mais complexo e mais dependente de conhecimento prévio dos avaliadores. É possível observar, ainda, que a quantidade total de critérios propostos pelas abordagens é, em sua maioria, bastante inferior ao total de critérios identificados para os modelos de referência adotados como base para este estudo (60 critérios) indicando, de antemão, fragilidade na abrangência das proposições. Outro fator relevante diz respeito à diferença na quantidade de critérios por dimensão: a qualidade de software é predominante para duas abordagens (Rocha e MAQSE), a qualidade de uso, para três abordagens (MAQSEI, MAEP e FASE), havendo uma predominância significativa para os critérios de qualidade pedagógica nas demais abordagens (Reeves, Mucchielli, TICESE, IAQSEM, Savi, ASE, Rodrigues e PETESE). Este cenário permite inferir que não existe uma abordagem que considere com igual relevância as três dimensões investigadas.

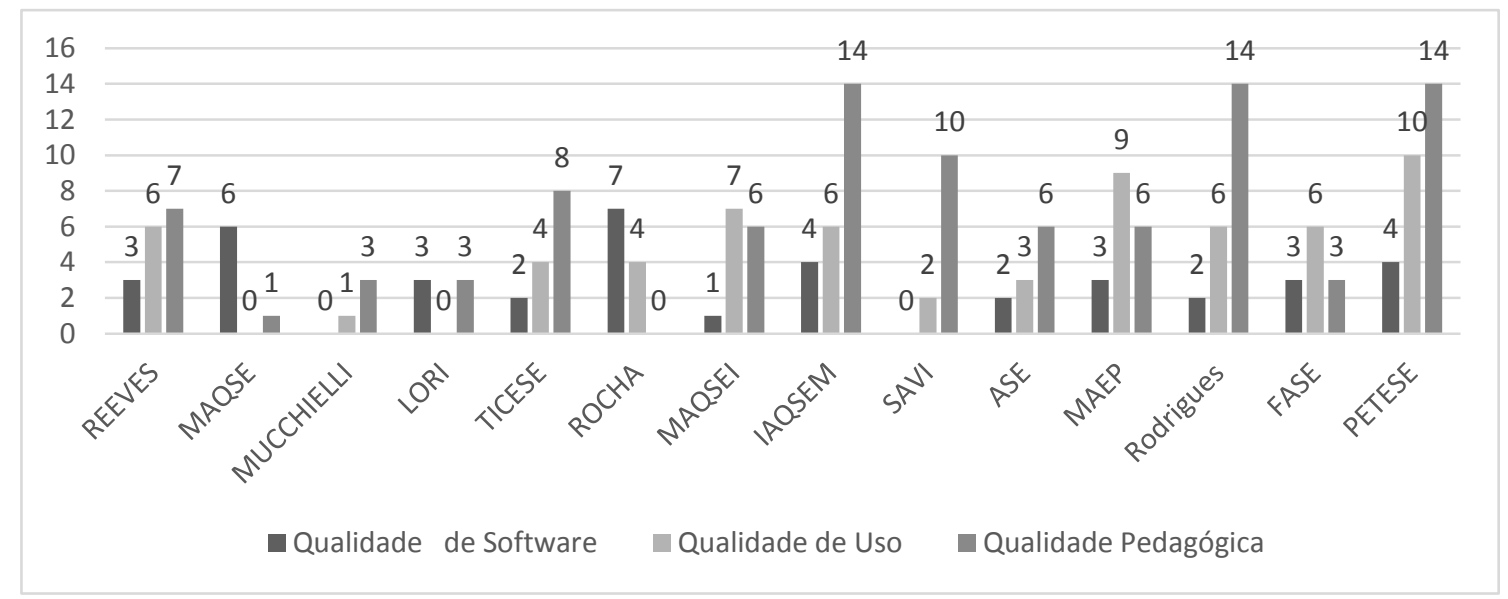

\section{Gráfico 1. Agrupamento de critérios por dimensão de qualidade}

Para os instrumentos de coleta de dados sugeridos nas abordagens, tem-se que metade destas utilizam escalas numéricas de avaliação (MAQSE, Muchielli, LORI, TICESE, Rocha, Savi e MAEP), cujas variações são de 3, 4, 5 ou 7 pontos; e a outra metade utiliza escalas não numéricas (Reeves, MAQSEI, IAQSEM, ASE, Rodrigues, FASE e PETESE). Este cenário reflete diretamente no tipo de resultado fornecido para análise e posterior diagnóstico sobre a avaliação. Os resultados podem ser valores percentuais (MAQSE, TICESE e Rocha), proporção de adequação aos critérios (MAQSEI, IAQSEM, ASE, Rodrigues, FASE e PETESE), análise de marcações em 
V Congresso Brasileiro de Informática na Educação (CBIE 2016)

Anais do XXVII Simpósio Brasileiro de Informática na Educação (SBIE 2016)

escalas bidirecionais (Reeves) ou, ainda, valores inteiros como grau de importância dos critérios (Muchielli, LORI, Savi e MAEP).

Com a caracterização das abordagens percebe-se que, além da variedade de abordagens para avaliação de SE, também existe diversidade sobre como estas abordagens foram configuradas (quantidade e natureza dos critérios, instrumentos de coleta de dados e diagnóstico oferecido). Este cenário evidencia a ausência de padronização e de linguagem utilizada entre os estudiosos do tema, dificultando, por exemplo, comparar a qualidade de SEs avaliados a partir de abordagens distintas.

O mapeamento dos critérios presentes nas abordagens de avaliação de SE definidos pelos modelos de referência de qualidade de software, de uso e pedagógica está disponível aqui, devido à restrição de espaço deste documento. Sua análise permitiu responder as duas perguntas de pesquisa definidas para este estudo, explanadas a seguir.

QP 01 - Quantos e quais critérios de cada abordagem correspondem aos critérios definidos pelos modelos de referências (QS QU e QP)? Dos 21 critérios de Qualidade de Software, 16 são contemplados por pelo menos uma das abordagens consideradas no estudo, sendo os mais frequentes: Adaptabilidade e Apreensibilidade, com 6 e 5 ocorrências, respectivamente. A abordagem que mais contempla critérios de QS é o Método Rocha, com 7 critérios $^{1}$, sendo, portanto, a mais abrangente. Vale considerar que as abordagens de Muchielli e SAVI não contemplam nenhum critério de QS. A baixa adequação das abordagens ao modelo de qualidade de software pode ser associada ao fato deste abordar critérios técnicos de implementação do software, que muitas vezes não podem ser analisados por quem aplica as abordagens para avaliação de SE (professores). Todos os critérios de Qualidade de Uso (12) são contemplados por pelo menos uma das abordagens, sendo os mais frequentes: Ajuda e Documentação (7), Design Estético e Minimalista (9), Flexibilidade e Eficiência de Uso (9) e Liberdade e Controle ao Usuário (9). A abordagem que mais contempla critérios de QU é a PETESE, com 10 critérios $^{2}$, sendo a mais abrangente para esta dimensão. No entanto, as abordagens MAQSE e LORI não contemplam nenhum critério de QU. Este cenário revela uma conformidade das abordagens ao modelo de qualidade de uso, evidenciando a importância de considerar a boa usabilidade de softwares dessa natureza. Considerando os 27 critérios de Qualidade Pedagógica, 23 são contemplados por pelo menos uma das abordagens de avaliação de SE, sendo os critérios mais recorrentes: Atratividade para despertar o interesse de uso (9), Adequação do conteúdo ao públicoalvo (7), Adequação do SE ao conteúdo (6) e Favorece o trabalho em grupo, mas também pode ser utilizado individualmente (6). As abordagens que mais contemplam critérios de QP são IAQSEM ${ }^{3}$, Rodrigues ${ }^{4}$ e PETESE $^{5}$, com 14 critérios cada, sendo,

\footnotetext{
1 Intelegibilidade, Apreensibilidade, Operacionalidade, Tempo, Analisabilidade, Conformidade e Substituibilidade.

${ }^{2}$ Visibilidade do Estado do Sistema, Liberdade e Controle ao Usuário, Prevenção de Erros, Reconhecer em Vez de Relembrar, Flexibilidade e Eficiência de Uso, Design Estético e Minimalista, Suporte para o Usuário Reconhecer, Diagnosticar e Recuperar Erros, Ajuda e Documentação, Adaptabilidade, Compatibilidade.

${ }^{3}$ Atratividade para despertar o interesse de uso, Desafios pedagógicos para manter o nível de interesse, Interação com o usuário que estimule a participação e auxilie na superação de conflitos cognitivos, Adequação dos recursos de imagem e animação às atividades pedagógicas, Consistência pedagógica, Adequação do SE ao conteúdo, Adequação do conteúdo ao público-alvo, Retrabalho com os conhecimentos prévios, Guia de apoio pedagógico, Identificação dos objetivos pedagógicos,
} 
portanto a mais abrangente para esta dimensão. Vale considerar que todas as abordagens contemplam pelo menos um critério de QP. Esta aderência revela-se coerente, pois o proposito destas abordagens é a avaliação de SE, e por seu objetivo pedagógico devem considerar predominantemente este aspecto ao avaliar o SE.

QP 02 - Quantos e quais dos critérios dos modelos de referência ( $Q S Q U$ e $Q P$ ) não foram contemplados por nenhuma das abordagens? Do modelo de Qualidade de Software, 5 critérios não foram contemplados por nenhuma abordagem investigada, a saber: acurácia, conformidade funcional, maturidade, tolerância à falhas e modificabilidade; para o modelo de Qualidade Pedagógica; 4 critérios não possuem correspondente às abordagens investigadas: correção das simplificações do conteúdo, atualidade da metodologia, indicação dos conhecimentos prévios e dar ênfase a uma disciplina específica. $\mathrm{O}$ fato de poucos critérios dos modelos de qualidade da literatura não serem contemplados por nenhuma abordagem indica que, de uma forma geral, os modelos podem ser quase que totalmente contemplados em uma avaliação caso exista uma combinação das abordagens adotadas.

\section{Considerações Finais}

Considerando a importância da adequada adoção de recursos de informática dentro e fora da sala de aula, especificamente Softwares Educativos, e entendendo que estes recursos devem ser adequados pedagogicamente, corretos funcionalmente $\mathrm{e}$ direcionados às necessidades e habilidades de seus usuários, assume-se como pressuposto que as abordagens para avaliação de SE devem considerar os aspectos de Qualidade de Software, de Uso e Pedagógica. Como resultado do trabalho de pesquisa tem-se que não existe uma abordagem que contemple com igual relevância os três aspectos investigados, destacando fragilidade na coerência das abordagens em relação aos modelos de qualidade apresentados na literatura. Além disso, identificou-se a necessidade de padronização das abordagens analisadas.

A partir deste estudo, também foi possível identificar uma grande diversidade entre critérios e ferramentas para coleta de dados, o que pode comprometer a utilização destas na avaliação de SE. Outro ponto observado é a correlação de critérios distintos

\footnotetext{
Apresentação de uma abordagem interdisciplinar, Emite feedback encorajador e isento de carga negativa, Favorece a interpretação do estudante sobre seus erros e acertos, Permite o registro de desempenho do estudante.

${ }^{4}$ Atratividade para despertar o interesse de uso, Desafios pedagógicos para manter o nível de interesse, Adequação dos recursos de imagem e animação às atividades pedagógicas, Consistência pedagógica, Adequação do SE ao conteúdo, Utilização do SE como ferramenta didática, Adequação do conteúdo ao público-alvo, Adequação do conteúdo ao currículo escolar, Retrabalho com os conhecimentos prévios, Identificação de objetivos pedagógicos, Apresenta uma abordagem interdisciplinar, Emite feedback encorajador e isento de carga negativa, Favorece a interpretação do estudante sobre seus erros e acertos, Favorece o trabalho em grupo, mas também pode ser utilizado individualmente.

5 Atratividade para despertar o interesse de uso, Desafios pedagógicos para manter o nível de interesse, Adequação dos recursos de hipertexto às atividades pedagógicas, Adequação dos recursos de imagem e animação às atividades pedagógicas, Correção da representação do conteúdo, Adequação do conteúdo ao público-alvo, Adequação do conteúdo ao currículo escolar, Identificação dos objetivos pedagógicos, Possui diferentes graus de complexidade de conteúdo nas atividades, Apresenta uma abordagem interdisciplinar, Emite feedback encorajador e isento de carga negativa, Favorece a interpretação do estudante sobre seus erros e acertos, Permite o registro de desempenho do estudante, Favorece o trabalho em grupo, mas também pode ser utilizado individualmente.
} 
V Congresso Brasileiro de Informática na Educação (CBIE 2016)

Anais do XXVII Simpósio Brasileiro de Informática na Educação (SBIE 2016)

diante de uma mesma classificação. Entende-se como limitação desta pesquisa o fato de o estudo ter sido desenvolvido apenas por um aluno; no entanto, para minimizar a limitação o mapeamento foi revisado por dois professores com experiência nas áreas de IHC, Qualidade de Software e Avaliação de SE. Além disto, pretende-se, como trabalho futuro, desenvolver um dicionário de termos para facilitar a interpretação de critérios; o dicionário irá apresentar os critérios das abordagens que apresentam correspondências com os modelos de qualidade.

\section{Referências}

Atayde, A. P.R. "Metodologia de Avaliação de Qualidade de Software Educacional Infantil - MAQSEI". 2003. 250 f. Dissertação (Mestrado). Universidade Federal de Ciências da Computação, Belo Horizonte, MG, Brasil, 2003.

Bastien, C. and Scapin, D (1993). RT-0156 - Ergonomic criteria for the evaluation of human-computer interfaces. Rapport technique de l'INRIA.

Campos, G. H. B (1994). "Metodologia para avaliação da qualidade de software educacional". Diretrizes para desenvolvedores e usuários. Rio de Janeiro: Coppe.

Coomans, Stéphanie and Lacerda, Gilberto Santos (2015). "PETESE, a Pedagogical Ergonomic Tool for Educational Software Evaluation".Procedia Manufacturing, v. 3, [p. 5881-5888], 2015.

Da Silva, A. C. B. and De França, R. S. (2014) "Avaliação de software educativo na formação docente: articulando teoria e prática". In: Anais do III Congresso Brasileiro de Informática na Educação (CBIE). XX Workshop de Informática na Escola (WIE). Dourados, Brasil.

Da Silva, A. C. B., De França, R. S. and Da Silva, W. C. (2011) "Uma proposição de critérios para avaliação de softwares educativos de Língua Portuguesa". In: Anais do XXII Simpósio Brasileiro de Informática na Educação (SBIE), Aracaju, Brasil.

De França, R. S., and Da Silva, A. C. B. (2014) "Avaliação de softwares educativos para o ensino de Língua Portuguesa". Revista Brasileira de Informática na Educação (RBIE), V. 22, N. 03, P. 23.

De Oliveira, B. J. O, and Aguiar, Y. P. C. (2014) "Análise de abordagens objetivas para avaliação de softwares educativos". In: Proceedings of the 13th Brazilian Symposium on Human Factors in Computing Systems. Foz do Iguaçu-PR, 2014.

Gladcheff, A. P, Zuffi, E. M and Silva, M. D. (2001). "Um Instrumento para Avaliação da Qualidade de Softwares Educacionais de Matemática para o Ensino Fundamental". VII Workshop de Informática na Escola, Fortaleza-CE, 2001.

Gamez, L. (1998). "TICESE - Técnica de inspeção de conformidade ergonômica de software educacional". 1998. Dissertação (Mestrado). Universidade do Minho, Portugal.

Godoi, Katia Alexandra de and Padovani, Stephania (2011). "Instumentos avaliativos de software educativo: uma investigação de sua utilização por professores". Estudos em Design (Revista online). Rio de Janeiro, V.19, N.1, P. 1-23.

ISO/IEC 9126-1 (2003). Software engineering - Product quality - Part 1: Quality model. In: Associação Brasileira de Normas Técnicas, 2003. 
V Congresso Brasileiro de Informática na Educação (CBIE 2016)

Anais do XXVII Simpósio Brasileiro de Informática na Educação (SBIE 2016)

Muchielli, Alex (1987). "L`enseignement par ordinateur". Paris: Presses Universitaires de France. Que sais-je?.

Nesbit, John C., Belfer, Karen and Leacock, Tracey (2003). "Learning object review instrument (LORI). E-learning research and assessment network", Disponível em: $<$ http://www.transplantedgoose.net/gradstudies/educ892/LORI1.5.pdf $>$. Acesso em: 18 abr. 2015.

Nielsen, J (1994). "Usability engineering". San Francisco: Morgan Kaufman.

Oliveira, C.C, Costa, J.W and Moreira, M (2001). Ambientes informatizados de aprendizagem. Produção e Avaliação de Software Educativo. Campinas: Papirus.

Peixoto, D. C. C., Campos, S. T., Resende, R. F. (2015) "Avaliação de Jogos Educacionais Multiusuários: Uma Revisão Sistemática da Literatura," Anais do XXVI Simpósio Brasileiro de Informática na Educação (SBIE), Maceió, Brasil.

Pressman, R. (1997)."Software engineering: a practitioner's approach". McGraw-Hill: New York.

Reeves, T. (1994). "Systematic Evaluation Procedures for Interactive Multimedia for Education and Training" .In: Multimedia Computing: preparing for the 21 st century. Harrisburg, PA: Idea Group.

Rocha, A. R. and Campos, G. H. B (1993). Avaliação da Qualidade de Software Educacional. Brasília: Em aberto.

Rodrigues, G. C. F. S (2014). "Instrumento para avaliação de jogos eletrônicos educativos do Ensino Fundamental I". Dissertação (Mestrado Profissional em Linguística e Ensino). Universidade Federal da Paraíba, João Pessoa.

Savi, Rafael (2010). "Proposta de um modelo de avaliação de jogos educacionais". RENOTE, v. 8, n. 3.

Silva, Ana Cristina Barbosa da (2012)."Softwares Educativos: Critérios de Avaliação a partir dos Discursos da Interface, da Esfera Comunicativa e do Objeto de Ensino". 2012. Tese de Doutorado. Universidade Federal de Pernambuco, Centro de Educação. Programa de Pós-Graduação em Educação.

Silva, Cassandra Ribeiro de Oliveira (2002). "MAEP: um método ergopedagógico interativo de avaliação para produtos educacionais informatizados". Tese de Doutorado. Universidade Federal de Santa Catarina, Centro Tecnológico. Programa de Pós-Graduação em Engenharia de Produção.

Shneiderman, B. and Plaisant, C (2004). Designing the user interface: strategies for effective human-computer interaction.4. ed. Addison-Wesley Publishing Company.

Swebok (2004)."Guide to the Software Engineering Body of Knowledge".Version Project of the IEEE Computer Society Professional Practices Committee.

Vieira, F. M. S (1999). "Avaliação de Software Educativo: Reflexões para uma Análise Criteriosa", Centro Interdisciplinar de novas Tecnologias na Educação. Rio Grande do Sul.

Webber, Carine, Boff, Elisa and Bono, Fernanda (2009). "Ferramenta Especialista para Avaliação de Software Educacional". In: XX Simpósio Brasileiro de Informática na Educação. Florianópolis, SBIE/SBC, 2009. 